\title{
Retrospective Evaluation
}

\section{e Percutaneous Endoscopic Lumbar Discectomy for L5-S1 Disc Herniation: Consideration of the Relation between the Iliac Crest and L5-S1 Disc}

\author{
Kyung-Chul Choi MD, $\mathrm{PhD}^{1}$, and Choon-Keun Park MD, PhD²
}

From: ${ }^{1}$ Department of Neurosurgery, Leon Wiltse Memorial Hospital, Anyang, Korea; '2Department of Neurosurgery, Leon Wiltse Memorial Hospital, Suwon, Korea

Address Correspondence: Kyung-Chul Choi MD, PhD Department of Neurosurgery, Leon Wiltse Memorial Hospital, Anyang, Korea 560 Gyeongsu-daero Dongan-gu, Anyang-si

Gyeongi-do, 431-841 Republic of Korea

E-mail: chul5104@hanmail.net

Disclaimer: There was no external funding in the preparation of this manuscript. Conflict of interest: Each author certifies that he or she, or a member of his or her immediate

family, has no commercial association (i.e., consultancies, stock ownership, equity interest, patent/licensing arrangements, etc.) that might pose a conflict of interest in connection with the submitted manuscript.

Manuscript received: 05-22-2015 Accepted for publication: 08-06-2015

Free full manuscript: www.pain physicianjournal.com
Background: Percutaneous transforaminal techniques for the treatment of lumbar disc herniation have markedly evolved. Percutaneous endoscopic lumbar discectomy (PELD) for L5-S1 disc herniation is regarded as challenging due to the unique anatomy of the iliac crest, large facet joint, and inclinatory disc space. Among these, the iliac crest is considered a major obstacle. There are no studies regarding the height of the iliac crest and their appropriate procedures in PELD.

Objectives: This study discusses PELD for L5-S1 disc herniation and the appropriate approach according to the height of iliac crest.

Study Design: Retrospective evaluation.

Methods: 100 consecutive patients underwent PELD via the transforaminal route for L5-S1 disC herniation by a single surgeon. The study was divided into 2 groups: the foraminoplasty group requiring foraminal widening to access the herniated disc and the non-foraminoplasty group treated by conventional posterolateral access. Radiological parameters such as iliac height, the relative position of the iliac crest to the landmarks of the L5-S1 level, iliosacral angle and foraminal height, and disc location were considered. Clinical outcomes were assessed by the Visual Analogue Scale (VAS, 0 - 10) for back and leg pain, the Oswestry Disability Index (ODI, $0-100 \%$ ), and the modified MacNab criteria.

Results: The overall VAS scores for back and leg pain decreased from 6.0 to 2.3 and from 7.5 to 1.7. The mean ODI (\%) improved from 54.0 to 11.6. Using modified MacNab criteria, a good outcome was $92 \%$. Foraminoplasty was required in 19 patients. lliac crest height was significantly higher in the foraminoplasty group than the non-foraminoplasty group $(37.7 \mathrm{~mm}$ vs $30.1 \mathrm{~mm}$, $P<0.001)$. In the foraminoplasty group, the iliac crest is above the mid L5 pedicle on lateral radiography in all cases. There were no significant differences in foraminal height, foraminal width, iliosacral angle, or disc height between the 2 groups. In addition, there were no differences in clinical outcome between the 2 groups.

Limitations: This study is a retrospective analysis and simplifies the complexity of the L5-S1 level and iliac bone using two-dimensional radiography.

Conclusion: In high iliac crest cases where the iliac crest is above the mid L5 pedicle in lateral radiography, foraminoplasty may be considered for transforaminal access of L5-S1 disc herniation. Conventional transforaminal access can be utilized with ease in low iliac crest cases where the iliac crest is below the mid-L5 pedicle.

Key words: Percutaneous endoscopic lumbar discectomy, transforaminal, L5-S1, iliac crest, foraminoplasty

IRB Number: 2015-W05

Pain Physician 2015; 18:E301-E308 
T he concept of percutaneous discectomy has been changed from decompression of the central nucleus to removal of the extruded disc fragment. Percutaneous endoscopic lumbar discectomy (PELD) has markedly evolved since posterolateral endoscopic discectomy was introduced in 1987 (1). There have been several reports about transforaminal percutaneous endoscopic lumbar discectomy but few reports discuss the L5-S1 level $(2,3)$. Endoscopic lumbar discectomy is performed via 2 routes: transforaminal and interlaminar. The 2 techniques are distinct in the involved surgical anatomy and utilized instruments. The surgical route depends on several variables: relative placement of the iliac bone and L5-S1 disc space, disc location, and surgeon's preference (2). Conventionally, transforaminal PELD is accepted from L1-2 to L4-5 levels and interlaminar PELD is accepted at the L5-S1 level $(4,5)$. Many authors suggest that high iliac crest is an obstacle in transforaminal PELD at the L5-S1 level and even at the L4-5 level (4-6). But, definition of high iliac crest is vague and there are no studies concerning the difficulty of transforaminal PELD in certain situations for L5-S1 disc herniation.

Here, the authors describe their experience of transforaminal PELD at the L5-S1 level. They elucidate the appropriate height of the iliac crest for PELD at the L5-S1 level.

\section{Methods}

This present study was approved by our Institutional Review Board (2015-W05). One hundred consecutive patients were treated by transforaminal PELD for L5-S1 disc herniation between July 2011 and December 2013 by a single surgeon (KC Choi). The inclusion criteria were (1) intra-canal disc herniation, (2) radicular leg pain or back pain associated with disc herniation, and (3) failure of conservative treatments over 6 weeks. The exclusion criteria were (1) foraminal or extraforaminal disc herniation, (2) definite congenital anomalies, including lumbarization, (3) spondylolysis, and (4) instability. Pre- and postoperative data were obtained from a chart review and radiological examination including magnetic resonance imaging (MRI) and computed tomography (CT). An independent observer performed the radiological assessments. The location of herniation in relation to the pedicle and spinal canal was described either as central or paramedian. A higher herniation midline split ratio exceeding 60:40 placed the herniation in the para- median group. Disc height was defined as the vertical distance between the posterior lower plate of the $L 5$ vertebral body (VB) and the posterior upper plate of the sacrum. Foraminal height was defined as the vertical distance from the lower margin of the L5 pedicle to the posterior lower endplate of L5 VB. Foraminal width was defined as the widest horizontal distance between the posterior vertebral line of $L 5 \mathrm{VB}$ and the superior facet of the $\mathrm{S} 1$ vertebra. Iliac height was defined as the vertical distance between the tangential line of the iliac crest and the superior medial point of the S1 pedicle in anterior posterior radiography. The iliosacral angle was defined as the angle between the tangential line of the iliac crest and the line from the highest point of the iliac crest and the superior-medial point of the S1 pedicle. In lateral radiography, the location of the highest point of the iliac crest was classified using the bony landmark (Fig. 1). Type 1 is that the highest point of the iliac crest is below the upper endplate of the sacrum. Type 2 is the point between the lower endplate of L5 VB and the upper endplate of the sacrum. Type 3 is the point between the lower margin of the $L 5$ pedicle and the lower endplate of L5 VB. Type 4 is the point between the mid-point of the $L 5$ pedicle and the lower margin of the $L 5$ pedicle. Type 5 is the point between the upper margin of the L5 pedicle and the mid-point of the L5 pedicle. Type 6 is the point between the upper margin of the L5 pedicle and the lower endplate of L4 VB. Clinical and functional outcomes were assessed using a visual analogue scale (VAS, $0-10)$ and the Oswestry Disability Index (ODI, $0-100 \%$ ), respectively. Clinical outcomes were also assessed by the Modified MacNab criteria (Excellent: no pain, no restriction of mobility, return to normal work and level of activity; Good: occasional nonradicular pain, relief of presenting symptoms, able to return to modified work; Fair: some improved functional capacity, still handicapped and/or unemployed; Poor: continued objective symptoms of root involvement, additional operative intervention needed at the index level irrespective of the length of postoperative follow-up). The present study was divided into a foraminoplasty group that requires foraminal widening, and a non-foraminoplasty group that uses conventional posterolateral access.

\section{Statistical Analysis}

All statistical analyses were performed using $R$ for Windows (version 3.1.2). Intergroup differences were analyzed using Fisher's exact test, the chi-square test, 


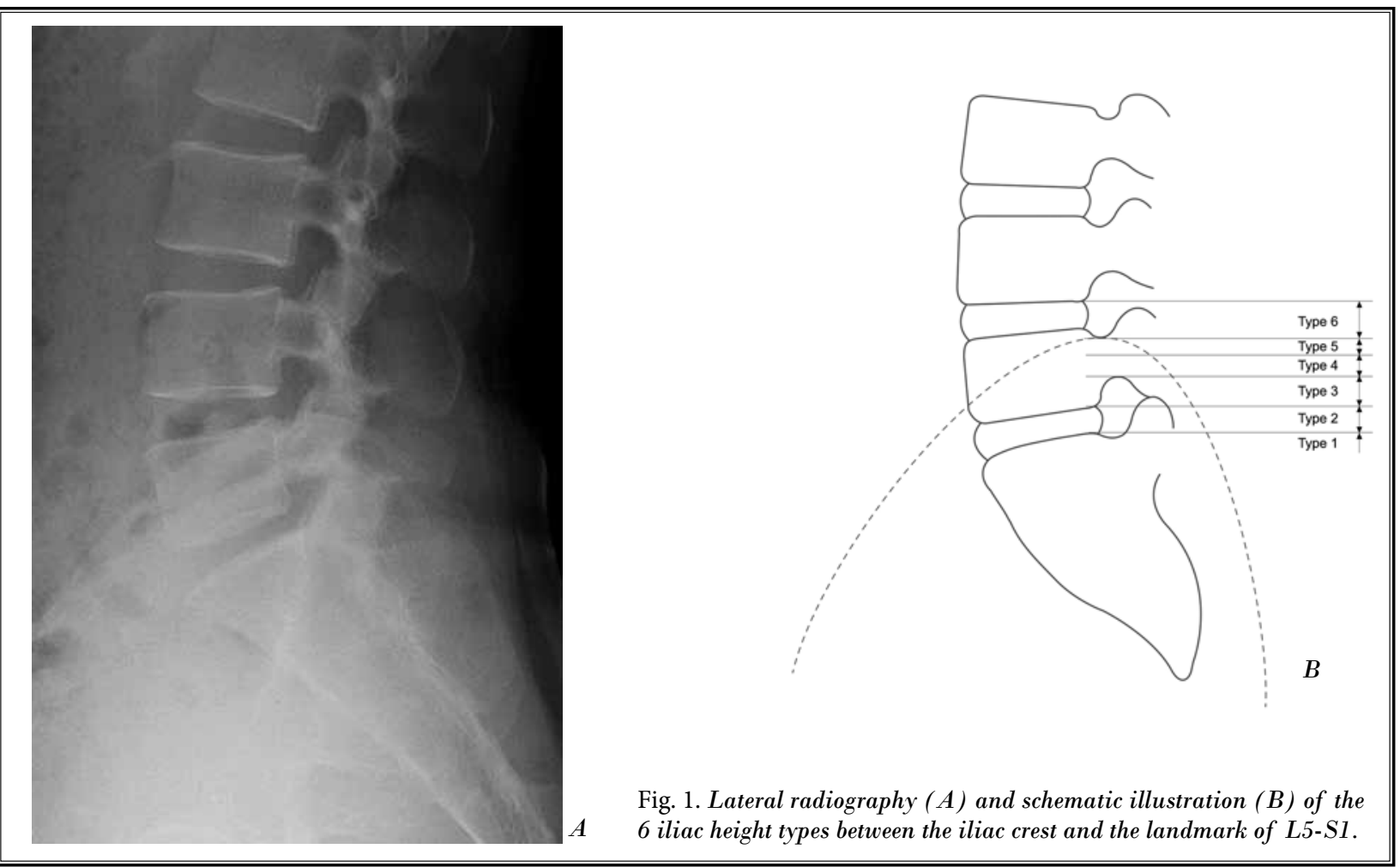

independent t-test, or Mann-Whitney U-test where appropriate. The level of significance was set at $P<$ 0.05 .

\section{PELD Technique}

The PELD procedure was performed under local anesthesia in the prone position. The skin entry point was generally superior to the iliac crest and $10-13 \mathrm{~cm}$ from the midline. After infiltration of the entry point with local anesthetics, an 18-gauge spinal needle was introduced, under the guidance of fluoroscopic imaging. The final target point of the spinal needle was the medial pedicular line on the anteroposterior (AP) image and the posterior vertebral line on the lateral image. When the targeted disc was a central disc herniation, the spinal needle was targeted more towards the medial pedicular line on the AP view and the posterior vertebral line on the lateral view. Subsequently, an epidurography was performed using contrast media to confirm the location of the exiting and traversing root. However, if the spinal needle was on the medial pedicular line on the AP view and the needle tip was located within the disc space on the lateral view, foraminoplasty was performed. Before the needle was withdrawn, a guide wire was inserted to introduce the serial dilation. Using the bone cutter
(Arthro Kinetics PLC, Park Green, Macclesfield, U.K.), the tip of the superior facet was undercut (Fig. 2). The bone cutter could be advanced to the medial pedicular line under fluoroscopy. After foraminal widening, needling was retried. Checking the location of the spinal needle was needed, which was the medial pedicular line on the AP image and the posterior vertebral line on the lateral image. After inserting the spinal needle into the disc, the nucleus pulposus was stained blue with $1 \mathrm{~mL}$ contrast media (Telebrix, Guerbet, France) and indigo carmine (Carmine, Korea United Pharmaceutical, Yoenki, Korea) solution for discography. The following steps were then performed: a guide wire was inserted through the spinal needle; the spinal needle was removed; a small incision was made in the skin at the entry site; a tapered cannulated obturator was inserted along the guide wire; after touching the annulus, the obturator was inserted into the disc by hammering; and lastly, a bevel-ended, oval-shaped working cannula was inserted into the disc along the obturator, after which the obturator was removed. Next, an endoscope (YESS system; Richard Wolf GmbH, Germany) was inserted through the cannula. The pathological nucleus was stained for easy discrimination. The blue-stained disc was removed using endoscopic forceps and a side- 


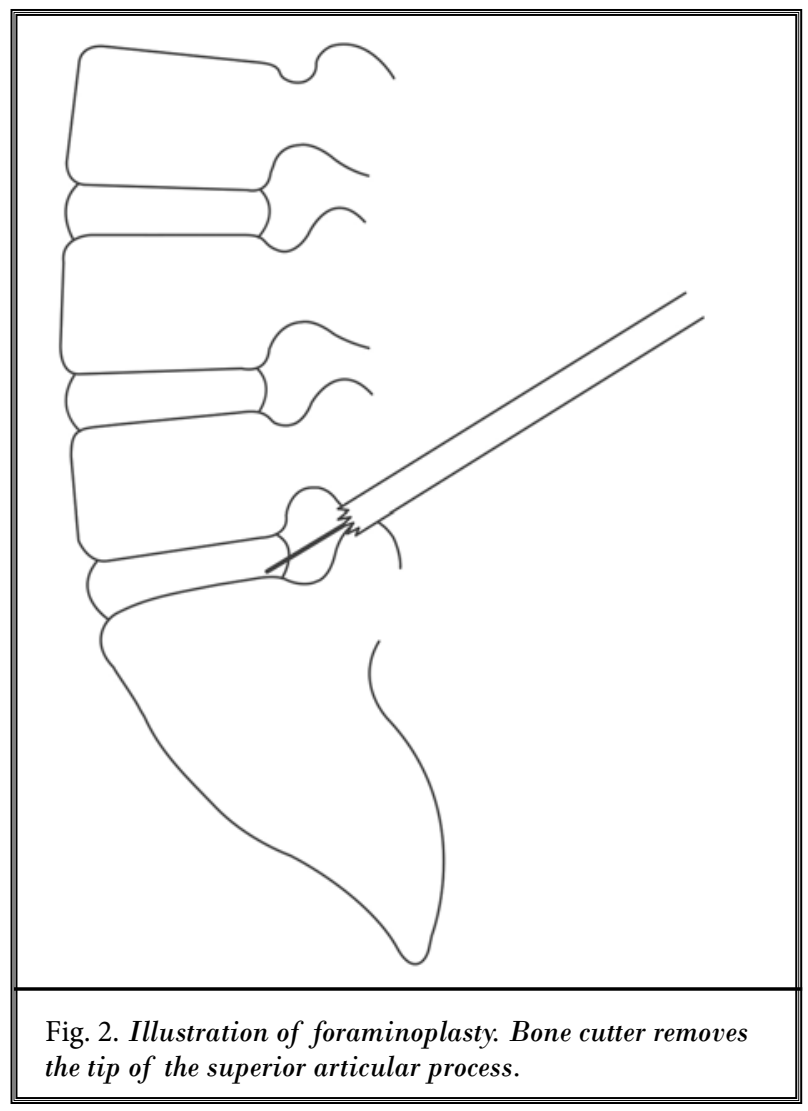

firing holmium:yttrium-aluminum-garnet (Ho:YAG) laser. The inflamed nucleus was observed as being anchored by the annular fissure. The herniated disc and fibrotic scar tissues were released and removed using endoscopic forceps and a side-firing Ho:YAG laser. After the herniated fragment was completely removed, the endoscope was removed and a sterile dressing was applied with a one point suture.

\section{Results}

One hundred consecutive patients underwent PELD for L5-S1 disc herniation via the transforaminal route. The mean age of the patients was $36.5 \pm 11.3$ years old. There were 49 men and 51 women. The overall VAS score for back and leg pain decreased from $6.0 \pm$ 1.9 to $2.3 \pm 0.9$ and $7.5 \pm 1.4$ to $1.7 \pm 1.3$. The mean ODI (\%) improved from $54.0 \pm 16.6$ to $11.6 \pm 9.0$. According to the modified MacNab criteria, the outcomes rated as excellent were $65(65 \%)$, good $27(27 \%)$, fair 5 (5\%), and poor $3(3 \%)$. The mean time to return to work was $3.2 \pm 1.4$ weeks. Incomplete removal of disc fragments was observed in 2 patients. During the 2-year followup, disc herniation recurred in 2 cases. There were no cases of postoperative dysesthesia caused by L5 nerve root injury or dural tear. Foraminoplasty was required in 19 patients. The height of the iliac crest was significantly higher in the foraminoplasty group than in the non-foraminoplasty group $(37.7 \pm 5.9 \mathrm{~mm}$ vs $30.1 \pm 9.0$ $\mathrm{mm}, P<0.001$; Table 1). In the foraminoplasty group, the iliac crest was above the mid L5 pedicle on lateral radiography in all cases. The iliac crests below the mid L5 pedicle did not require foraminoplasty in any cases $(P=0.001)$. There was no significant difference in the foraminal height, foraminal width, iliosacral angle, or disc height between the 2 groups. In addition, there were no differences in clinical outcomes between the 2 groups.

\section{Discussion}

Herniated disc in intracanal disc, migrated disc, foraminal/extraforaminal, and large disc herniation can be removed by PELD (7-9). But, at the L5-S1 level, PELD via a transforaminal route is challenging due to the obstructive anatomy. The iliac crest and the inclination of the L5-S1 level frequently obstruct transforaminal approach, leading to a steeper trajectory angle that reaches far from the extruded disc (10). The location and angle of the working channel are key aspects of successful PELD (11). At the lumbar level, a trajectory angle of more or less than $25^{\circ}$ is appropriate (12). Although the coronal angle is more than 30 degrees, it is enough to remove the herniated disc with a levering technique which lets the working cannula tilt down. However, at the L5-S1 level, the working cannula does not have leverage due to the narrow foramen and iliac crest. A lateral or horizontal approach provides direct visualization of the extruded disc and makes it far easier to remove disc fragments. Nevertheless it is impossible to perform an extreme lateral or lateral approach in L5-S1 disc herniation. Only access point is via suprailiac entry or transiliac entry, making a tunnel through the iliac crest $(2,4,13)$. Another concern is the large facet joint which causes the steep trajectory angle. This limitation often results in remnant disc fragments. The suprailiac approach passes the tip of the superior facet. The profile of superior facet resembles a tringle. This method reduces the approach angle and avoids irritating the exiting nerve root $(2,3)$.

Interlaminar PELD is usually used to remove a herniated disc at the L5-S1 level. The biggest difference between interlaminar (IL) PELD and transforaminal (TF) PELD is that in IL-PELD neural structures are encountered before the removal of the disc, while in TF-PELD, 
Percutaneous Endoscopic Lumbar Discectomy for L5-S1 Disc Herniation

Table 1. Radiographic and clinical comparison between the foraminoplasty group and the non-foraminoplasty group.

\begin{tabular}{|c|c|c|c|}
\hline & Foraminoplasty & Non-foraminoplasty & $P$ value \\
\hline No & 19 & 81 & \\
\hline Age & $38.3( \pm 10.3)$ & $36.1( \pm 11.5)$ & $0.386^{*}$ \\
\hline Gender $(\mathrm{M}: \mathrm{F})$ & $11(57.9 \%): 8(42.1 \%)$ & $38(46.9 \%): 43(53.1 \%)$ & $0.389 \ddagger$ \\
\hline Iliac height (mm) & $37.73( \pm 5.9)$ & $30.11( \pm 9.0)$ & $0.000^{*}$ \\
\hline \multicolumn{3}{|l|}{ Iliac height grade } & \multirow{3}{*}{$0.001 ”$} \\
\hline Type $\geq 5$ & $19(100 \%)$ & $50(61.7 \%)$ & \\
\hline Type $<5$ & $0(0 \%)$ & $31(38.3 \%)$ & \\
\hline Iliosacral angle $\left({ }^{\circ}\right)$ & $25.6( \pm 5.6)$ & $23.9( \pm 6.1)$ & $0.519^{*}$ \\
\hline Disc height $(\mathrm{mm})$ & $8.0( \pm 2.6)$ & $8.5( \pm 2.0)$ & $0.324 \dagger$ \\
\hline Foraminal height $(\mathrm{mm})$ & $7.4( \pm 3.5)$ & $7.7( \pm 2.6)$ & $0.923^{*}$ \\
\hline Disc location (central : paramedian) & $2(10.5 \%): 17(89.5 \%)$ & $26(32.1 \%): 55(67.9 \%)$ & $0.087^{\prime \prime}$ \\
\hline Preop VAS back & $5.5( \pm 1.7)$ & $6.1( \pm 1.9)$ & $0.195^{*}$ \\
\hline preop VAS leg & $7.8( \pm 1.1)$ & $7.4( \pm 1.5)$ & $0.411^{*}$ \\
\hline preop ODI & $58.8( \pm 14.9)$ & $52.9( \pm 16.9)$ & $0.207^{\star}$ \\
\hline postop VAS back & $2.4( \pm 0.5)$ & $2.3( \pm 0.9)$ & $0.316^{*}$ \\
\hline postop VAS leg & $1.7( \pm 0.8)$ & $1.7( \pm 1.4)$ & $0.749^{*}$ \\
\hline postop ODI (\%) & $11.2( \pm 8.4)$ & $11.7( \pm 8.0)$ & $0.936^{*}$ \\
\hline Time return to work (weeks) & $3.0( \pm 1.7)$ & $3.2( \pm 1.3)$ & $0.285^{*}$ \\
\hline
\end{tabular}

* Mann-Whitney U test. $\dagger$ Independent t-test. $\ddagger$ Chi-square test. "Fisher’s exact test

neural structures are found to be decompressed after the removal of the disc. IL-PELD has a higher possibility of neural injury such as postoperative dysesthesia because it requires manipulation of the neural tissue (2).

According to our series, if the height of the iliac crest is located below the mid pedicle of L5, a conventional posterolateral approach can be carried out without difficulty. However, if the height of the iliac crest is above the mid pedicle of L5, an appropriate working channel location sometimes requires foraminal widening to remove the herniated mass. In particular, in the situation that the height of the iliac crest is above the L4-5 disc space, android pelvis, or central disc herniation, foraminoplasty is considered to be necessary (Fig. 3).

Various endoscopic tools such as a laser, bone cutter, reamer, and drill are used in foraminoplasty for foraminal stenosis, down migrated disc, and L5-S1 intracanal disc herniation. Knight et al (14) introduced laser-assisted foraminoplasty for treating low back pain. Choi et al (7) applied this method in foraminoplasty to remove the hidden downward migrated disc. Ahn et al (15) introduced foraminotomy using an endoscopic drill for foraminal stenosis, and Jasper et al (16) reported foraminoplasty in degenerative spondylolisthesis. In these situations, a large amount of bone removal is necessary. The base of the superior facet joint, which is harder, should be removed. However, foraminoplasty when approaching L5-S1 disc herniation removes only a few superior parts of the superior facet joint with minimal time-consumption $(3,17)$. Neural injury and bone bleeding using a reamer blindly under fluoroscopic guidance may be a concern of physicians; however, working very close to the superior facet is safe and minimizes neural injury. Foraminal dimension decreases going down the levels of the spine. On the contrary, the window of safe working zone is widest at the L5-S1 level because the distance from the exiting nerve root to the margin of the disc and to the edge of superior articular process is farthest (18). The L5-S1 level has a relatively shorter disc height and larger facet joints. Widening of the posterior border (superior articular process) of the working zone facilitates entry into the epidural space. The ligamentum flavum also protects neural tissue from direct injury. A little undercutting of the superior facet rarely causes bleeding. A posterolateral endoscopic approach is effective in paramedian disc herniation and low-grade downward migrated disc herniation (Fig. 4). However, this approach is limited in disc fragment removal in upward migrated disc her- 

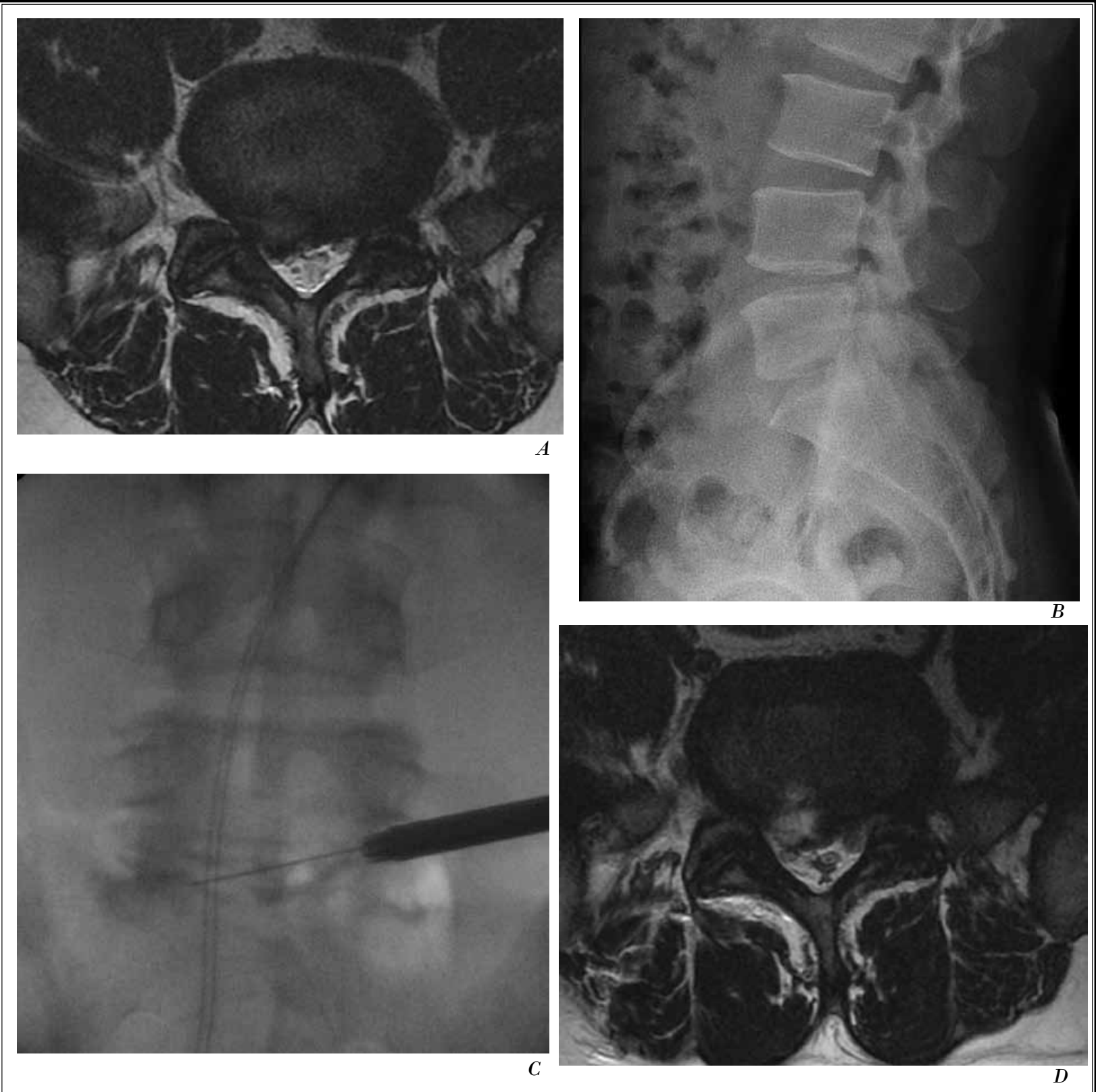

Fig. 3. A 47-year old man presented with right posterior radiating leg pain. Magnetic resonance image (MRI) (A) showing a paramedian extruded disc compressing S1 nerve root at the L5-S1 level. The top point of the iliac crest is located at the L4-5 disc space in lateral radiography $(B)$. Intraoperative radiography $(C)$ showing foraminoplasty, which undercut the superior articular process using a bone cutter. Postopertive MRI (D) showing removal of the extruded disc fragment.

niation and highly downward migrated disc herniation. A transiliac approach or interlaminar approach is rather preferred $(13,19,20)$. An inclined trajectory of a working cannula has the possibility of injury of the upper endplate of the $\mathbf{S 1}$ vertebra with a steep coronal angle. In advanced degenerative spine with facet joint hypertrophy, disc space narrowing, and osteophyte formation of the $\mathrm{VB}$, greater widening of the foramen is needed. Kubaszewski et al (21) introduced a transfacet resection technique with a guiding 

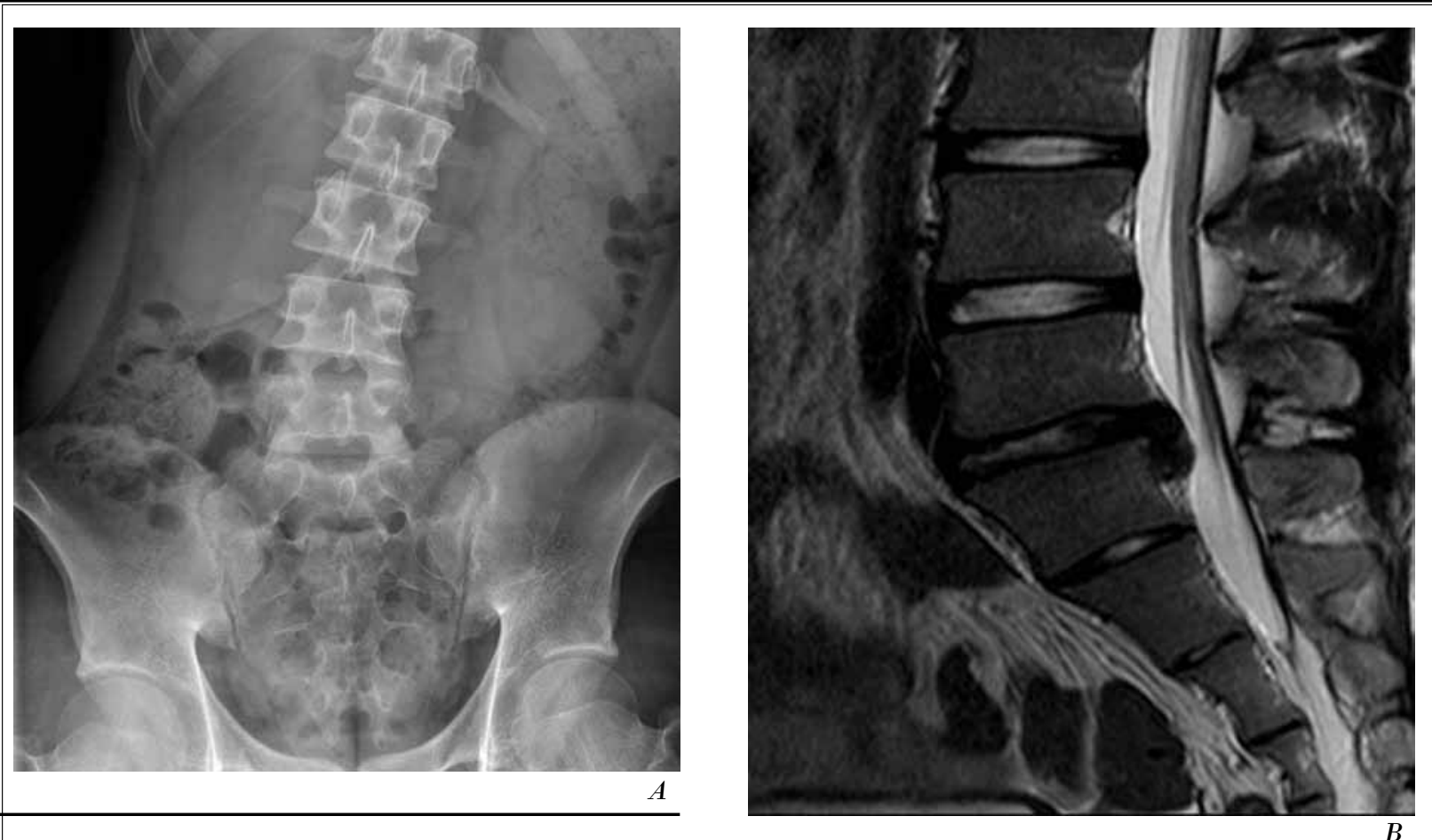

Fig. 4. A 19-year old girl presented with severe right leg pain.

Radiography $(A)$ showing the iliac crest below the $L 5$ pedicle with the sciatic list. Sagittal MRI (B) showing a downward migrated disc fragment at the L5-S1 level. After conventional transforaminal PELD, sagittal MRI (C) showing complete removal of the herniated disc.

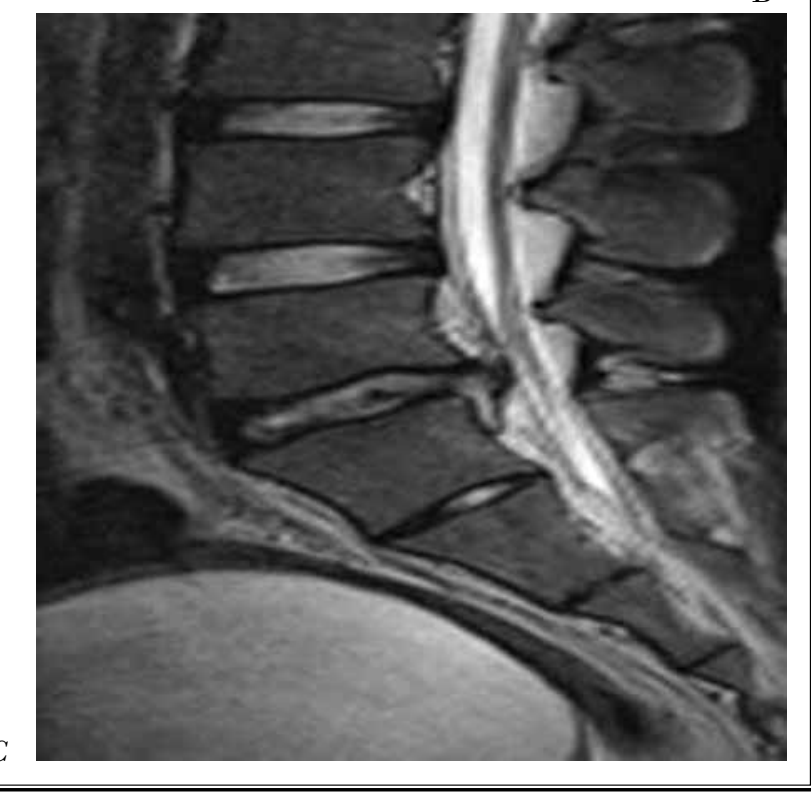

K-wire for degenerative L5-S1 level that needs a wide removal of facet.

This study is retrospective. A prospective study may provide clearer answers for this question. This study simplifies the complexity of the L5-S1 level and iliac bone by using a two-dimensional radiography. It needs further study using a three-dimensional method.

\section{Conclusion}

In high iliac crest cases, where the iliac crest is above the mid L5 pedicle in lateral radiography, foraminoplasty may be considered for transforaminal access for L5-S1 disc herniation. Transforaminal access can be utilized without difficulty in the low iliac crest where the iliac crest height is below the mid-L5 pedicle. 


\section{References}

1. Kambin P, Brager MD. Percutaneous posterolateral discectomy. Anatomy and mechanism. Clin Orthop Relat Res 1987; 223:145-154.

2. Choi KC, Kim JS, Ryu KS, Kang BU, Ahn $\mathrm{Y}$, Lee SH. Percutaneous endoscopic lumbar discectomy for $\mathrm{L}_{5}-\mathrm{S}_{1}$ disc herniation: Transforaminal versus interlaminar approach. Pain Physician 2013; 16:547-556.

3. Schubert M, Hoogland T. Endoscopic transforaminal nucleotomy with foraminoplasty for lumbar disk herniation. Oper Orthop Traumatol 2005; 17:641-661.

4. Lee $\mathrm{DH}$, Kim NH, Park JB, Hwang C), Lee CS, Kim YT, Kang SJ, Rhee JM. CT scan assessment of the pathway of the true lateral approach for transforaminal endoscopic lumbar discectomy: Is it possible? ] Bone Joint Surg Br 2011; 93:1395-1399.

5. Ruetten S, Komp M, Godolias G. An extreme lateral access for the surgery of lumbar disc herniations inside the spinal canal using the full-endoscopic uniportal transforaminal approachtechnique and prospective results of 463 patients. Spine (Phila Pa 1976) 2005; 30:2570-2578.

6. Ruetten S, Komp M, Merk H, Godolias G. Full-endoscopic interlaminar and transforaminal lumbar discectomy versus conventional microsurgical technique: A prospective, randomized, controlled study. Spine (Phila Pa 1976) 2008; 33:931-939.

7. Choi G, Lee SH, Lokhande P, Kong BJ, Shim CS, Jung B, Kim JS. Percutaneous endoscopic approach for highly migrated intracanal disc herniations by forami- noplastic technique using rigid working channel endoscope. Spine (Phila Pa 1976) 2008; 33: E508-E515.

8. Sasani M, Ozer AF, Oktenoglu T, Canbulat N, Sarioglu AC. Percutaneous endoscopic discectomy for far lateral lumbar disc herniations: Prospective study and outcome of 66 patients. Minim Invasive Neurosurg 2007; 50:91-97.

9. Lee SH, Choi KC, Baek OK, Kim HJ, Yoo $\mathrm{SH}$. Percutaneous endoscopic intra-annular subligamentous herniotomy for large central disc herniation: A technical case report. Spine (Phila Pa 1976) 2014; 39:E473-E479.

10. Lee SH, Kang BU, Ahn Y, Choi G, Choi YG, Ahn KU, Shin SW, Kang HY. Operative failure of percutaneous endoscopic lumbar discectomy: A radiologic analysis of 55 cases. Spine (Phila Pa 1976) 2006; 31:E285-E290.

11. Choi KC, Lee JH, Kim JS, Sabal LA, Lee $\mathrm{S}$, Kim H, Lee SH. Unsuccessful percutaneous endoscopic lumbar discectomy: A single-center experience of 10,228 cases. Neurosurgery 2015; 76:372-380.

12. Yeung AT, Tsou PM. Posterolateral endoscopic excision for lumbar disc herniation: Surgical technique, outcome, and complications in 307 consecutive cases. Spine (Phila Pa 1976) 2002; 27:722-731.

13. Choi G, Kim JS, Lokhande P, Lee SH. Percutaneous endoscopic lumbar discectomy by transiliac approach: A case report. Spine (Phila Pa 1976) 2009; 34:E443-E446.

14. Knight MT, Ellison DR, Goswami A, Hillier VF. Review of safety in endoscopic laser foraminoplasty for the management of back pain. J Clin Laser Med Surg 2001; 19:147-157.
15. Ahn Y, Oh HK, Kim H, Lee SH, Lee HN. Percutaneous endoscopic lumbar foraminotomy: An advanced surgical technique and clinical outcomes. Neurosurgery 2014; 75:124-133.

16. Jasper GP, Francisco GM, Telfeian AE. Transforaminal endoscopic discectomy with foraminoplasty for the treatment of spondylolisthesis. Pain Physician 2014; 17:E703-E708.

17. Lee SH, Kang HS, Choi G, Kong BJ, Ahn Y, Kim JS, Lee HY. Foraminoplastic ventral epidural approach for removal of extruded herniated fragment at the $\mathrm{L}_{5}$ Si level. Neurol Med Chir (Tokyo) 2010; 50:1074-1078.

18. Min JH, Kang SH, Lee JB, Cho TH, Suh JK, Rhyu IJ. Morphometric analysis of the working zone for endoscopic lumbar discectomy. J Spinal Disord Tech 2005; 18:132-135.

19. Osman SG, Sherlekar S, Malik A, Winters C, Grewal PK, Narayanan M, Gemechu N. Endoscopic trans-iliac approach to $L_{5}-S_{1}$ disc and foramen - a report on clinical experience. Int J Spine Surg 2014; 8 [Dec. 1 e-Collection].

20. Choi G, Prada N, Modi HN, Vasavada NB, Kim JS, Lee SH. Percutaneous endoscopic lumbar herniectomy for high-grade down-migrated $L_{4}-L_{5}$ disc through an $\mathrm{L}_{5}-\mathrm{S}_{1}$ interlaminar approach: A technical note. Minim Invasive Neurosurg 2010 Jun; 53:147-152.

21. Kubaszewski L, Kaczmarczyk J, Nowakowski A, Sulewski A. Foraminoplastic transfacet epidural endoscopic approach for removal of intraforaminal disc herniation at the $\mathrm{L}_{5}-\mathrm{S}_{1}$ level. Wideochir Inne Tech Malo Inwazyjne 2014; 9:96-100. 